\title{
Efeitos Cardiovasculares e Renais da Injeção Intra-Arterial de Contraste Radiológico lônico em Cães com Restrição Hídrica *
}

\section{Cardiovascular and Renal Effects of Intra-Arterial Injection of Ionic Radiological Contrast in Dogs under Fluid Restriction}

\author{
Marisa Aparecida Lima Verderese ${ }^{1}$; Pedro Thadeu Galvão Vianna TSA ${ }^{2}$,
} Yara Marcondes Machado Castiglia TSA ${ }^{2}$; Luiz Antonio Vane TSA ${ }^{2}$

\begin{abstract}
RESUMO
Verderese MAL, Vianna PTG, Castiglia YMM, Vane LA - Efeitos Cardiovasculares e Renais da Injeção Intra-Arterial de Contraste Radiológico lônico em Cães com Restrição Hídrica
\end{abstract}

JUSTIFICATIVA E OBJETIVOS: O objetivo desta pesquisa foi estudar os efeitos agudos do contraste radiológico em situações de restrição de volume, avaliando-se os efeitos renais e cardiovasculares após a injeção intra-arterial de contraste radiológico de alta osmolaridade.

MÉTODO: Participaram do estudo 16 cães anestesiados com tiopental sódico (15 mg. $\left.\mathrm{kg}^{-1}\right)$ e fentanil $\left(15 \mu \mathrm{g} \cdot \mathrm{kg}^{-1}\right)$ em bolus, seguido de infusão contínua nas doses de $40 \mu \mathrm{g} \cdot \mathrm{kg}^{-1} \cdot \mathrm{min}^{-1}$ (tiopental sódico) $0,1 \mu \mathrm{g} \cdot \mathrm{kg}^{-1} \cdot \mathrm{min}^{-1}$ (fentanil). Foi feita hidratação com solução de glicose a $5 \%\left(0,03 \mathrm{~mL} \cdot \mathrm{kg}^{-1} \cdot \mathrm{min}^{-1}\right)$ e a ventilação pulmonar foi controlada mecanicamente com ar comprimido. Foram verificados os seguintes atributos: freqüência cardíaca (FC); pressão arterial média $(P A M)$; pressão da veia cava inferior (PVI); débito cardíaco (DC); hematócrito (Ht); fluxo plasmático efetivo renal (FPER); fluxo sangüíneo renal (FSR); ritmo de filtração glomerular (RFG); fração de filtração; resistência vascular renal (RVR); volume urinário (VU); osmolaridade plasmática e urinária; depuração osmolar, depuração de água livre e depuração de sódio e de potássio; sódio e potássio plasmáticos; excreção urinária e fracionária de sódio e potássio e temperatura retal. Estes atributos foram avaliados em quatro momentos: 30 (M1), 60 (M2), 90 (M3) e 120 (M4) minutos após o início da infusão de para-aminohipurato de sódio e creatinina (início da experiência). No momento 2, no grupo $\mathrm{G} 1$ foi feita injeção intra-arterial de solução fisiológica a 0,9\% $\left(1,24 \mathrm{~mL}_{\mathrm{kg}} \mathrm{k}^{-1}\right)$, e no grupo $\mathrm{G} 2$ foi injetado contraste radiológico $\left(1,24 \mathrm{~mL} . \mathrm{kg}^{-1}\right)$ pela mesma via.

RESULTADOS: O grupo G1 apresentou aumento da FC, do FPER, do FSR, da osmolaridade plasmática, da depuração de sódio e da excreção urinária de sódio; apresentou ainda diminuição da osmolaridade urinária, do potássio plasmático, da depuração de potássio e da temperatura retal. No grupo G2 ocorreu aumento da FC, da RVR, do VU, da depuração osmolar, da depuração de sódio e da excreção urinária e fracionária de sódio; ocorreu também redução do (a): hematócrito, ritmo de filtração glomerular, fração de filtração,

\footnotetext{
* Recebido do (Received from) Departamento de Anestesiologia da Faculdade de Medicina de Botucatu - UNESP, Botucatu, SP

1. Pós-Graduanda do Departamento de Anestesiologia da Faculdade de Medicina da UNESP, Campus de Botucatu, nível de Doutorado

2. Professor Titular do CET/SBA do Departamento de Anestesiologia da

Faculdade de Medicina da UNESP, Campus de Botucatu
}

Apresentado (Submitted) em 17 de setembro de 2004 Aceito (Accepted) para publicação em 06 de janeiro de 2005

Endereço para correspondência (Correspondence to) Dr. Pedro Thadeu Galvão Vianna

Dept ${ }^{\circ}$ de Anestesiologia da FMB - UNESP

Distrito de Rubião Júnior

18618-970 - Botucatu, SP

(C) Sociedade Brasileira de Anestesiologia, 2005 osmolaridade urinária, depuração de água livre, sódio e potássio urinários, potássio plasmático e temperatura retal.

CONCLUSÕES: Neste estudo, conclui-se que a injeção intra-arterial do contraste radiológico causou efeito bifásico na função renal. Inicialmente, provocou aumento da diurese e da excreção de sódio, mas, posteriormente, houve piora das condições hemodinâmicas e, conseqüentemente, da função renal, com aumento da resistência vascular renal e diminuição do ritmo de filtração glomerular.

Unitermos: ANIMAIS: cães; MEIOS DE CONTRASTE; RIM: função; SISTEMA CARDIOVASCULAR: hemodinâmica

\section{SUMMARY}

Verderese MAL, Vianna PTG, Castiglia YMM, Vane LA - Cardiovascular and Renal Effects of Intra-Arterial Injection of Ionic Radiological Contrast in Dogs under Fluid Restriction

BACKGROUND AND OBJECTIVES: This study aimed at evaluating acute radiological contrast effects in fluid restriction situations, observing renal and cardiovascular effects after intra-arterial injection of high osmolarity radiological contrast.

METHODS: Participated in this study 16 dogs anesthetized with sodium thiopental (15 mg. $\left.\mathrm{kg}^{-1}\right)$ and fentanyl $\left(0.03 \mathrm{~mL} \cdot \mathrm{kg}^{-1}\right)$, followed by continuous infusion of $40 \mu \mathrm{g} . \mathrm{kg}^{-1} . \mathrm{min}^{-1}$ (sodium thiopental) and 0.1 $\mu \mathrm{g} \cdot \mathrm{kg}^{-1} \cdot \mathrm{min}^{-1}$ (fentanyl). Hydration was achieved with $5 \%$ glucose solution (0.03 mL. $\left.\mathrm{kg}^{-1} . \mathrm{min}^{-1}\right)$ and ventilation was mechanically controlled with compressed air. The following attributes were evaluated: heart rate $(H R)$; mean blood pressure (MBP); inferior vena cava pressure (IVP); cardiac output (CO); hematocrit (Ht); effective renal plasma flow (ERPF); renal blood flow (RBF); glomerular filtration rate (GFR); filtration fraction; renal vascular resistance (RVR), urinary volume (UV); plasma and urinary osmolarity; osmolar clearance; free water clearance; sodium and potassium clearance; plasma sodium and potassium; sodium and potassium urinary fractional excretion and rectal temperature. These attributes were evaluated in four moments: 30 (M1), 60 (M2), 90 (M3) and 120 (M4) minutes after sodium para-aminohipurate and creatinine administration (beginning of experiment). In moment 2 , $\mathrm{G} 1$ received intra-arterial $0.9 \%$ saline $\left(1.24 \mathrm{~mL} . \mathrm{kg}^{-1}\right)$ and $\mathrm{G} 2$ received intra-arterial radiological contrast $\left(1.4 \mathrm{~mL} . \mathrm{kg}^{-1}\right)$.

RESULTS: Group $G 1$ has presented increased HR, ERPF, RBF, plasma osmolarity, sodium clearance and sodium urinary excretion, in addition to decreased urinary osmolarity, plasma sodium, potassium clearance and rectal temperature. Group $\mathrm{G} 2$ has presented increased $H R, R V R$, UV, osmolar clearance, sodium clearance and sodium urinary and fractional excretion; there has also been decrease in hematocrit, glomerular filtration rate, filtration fraction, urinary osmolarity, free water clearance, urinary sodium and potassium, plasma potassium and rectal temperature.

CONCLUSIONS: This study has concluded that intra-arterial radiological contrast has promoted a two-phase effect on renal function. Initially it has promoted increased diuresis and sodium excretion but then the hemodynamic conditions impaired, and consequently renal function impaired, with increased renal vascular resistance and decreased glomerular filtration rate.

Key Words: ANIMALS: dogs; CARDIOVASCULAR SYSTEM; hemodynamic; CONTRAST MEDIA; KIDNEY: function 


\section{INTRODUÇÃO}

A administração de contraste radiológico continua a ser uma das causas iatrogênicas mais comuns de insuficiência renal aguda adquirida em hospital ${ }^{1-4}$. Embora a patogênese dessa condição permaneça pouco compreendida, parece ser devida a isquemia medular causada pela diminuição do fluxo sangüíneo renal ${ }^{1-4}$.

Para a prevenção de nefropatia por contraste radiológico foi recomendado o uso de fluidos por via venosa, manitol e furosemida, embora a eficácia desse método terapêutico não tenha sido comprovada ${ }^{5}$. Recentemente ${ }^{6}$, foi indicado o uso de acetilcisteína (150 mg. $\left.\mathrm{kg}^{-1}\right)$, infundida, por via venosa, 30 minutos antes do uso do contrate radiológico, e $50 \mathrm{mg} \cdot \mathrm{kg}^{-1}, 4$ horas após o procedimento radiológico. Ambas as doses de acetilcisteína foram diluídas em $500 \mathrm{~mL}$ de solução fisiológica. Os resultados deste tratamento mostraram que houve proteção renal contra a nefrotoxicidade causada pelo contraste radiológico ${ }^{7}$.

Na ocorrência de fatores predisponentes, a incidência de nefropatia por contraste radiológico pode alcançar valores bem maiores. Dentre os fatores, estão incluídos: desidratação, diabete melito, mieloma múltiplo, idade avançada, distúrbio cardíaco, uso de diuréticos, insuficiência renal e exames com contraste radiológico realizados em curto intervalo de tempo $^{2,8,9}$.

Durante a anestesia o contraste radiológico pode ser injetado pela via intra-arterial, com finalidade diagnóstica. Assim, é importante o conhecimento dos efeitos agudos e imediatos do contraste radiológico sobre as principais funções renais. O objetivo desta pesquisa foi estudar estes efeitos agudos do contraste radiológico em situações de restrição de volume. Com esta finalidade, o presente experimento foi realizado em cães sob restrição hídrica, sendo avaliados os efeitos imediatos renais e cardiovasculares após a injeção intra-arterial de contraste radiológico de alta osmolaridade.

\section{MÉTODO}

Após aprovação pela Comissão de Ética em Experimentação Animal da Faculdade de Medicina de Botucatu, UNESP, foram utilizados 16 cães adultos, machos, sem raça definida, com peso corpóreo variando de 7 a $17 \mathrm{~kg}$, fornecidos pelo $\mathrm{Bi}$ otério Central do "Campus" de Botucatu da Universidade Estadual Paulista. Foi feita indução anestésica com tiopental sódico $\left(15 \mathrm{mg} \cdot \mathrm{kg}^{-1}\right)$, fentanil $\left(15 \mu \mathrm{g} \cdot \mathrm{kg}^{-1}\right)$, e cloreto de alcurônio $\left(0,2 \mathrm{mg} \cdot \mathrm{kg}^{-1}\right)$, e a seguir infusão contínua de 40 $\mu \mathrm{g} \cdot \mathrm{kg}^{-1} \cdot \mathrm{min}^{-1}$ de tiopental sódico e $0,1 \mu \mathrm{g} \cdot \mathrm{kg}^{-1} \mathrm{~min}^{-1}$ de fentanil durante todo o período experimental. Após 30 minutos de infusão de para-aminohipurato de sódio $(\mathrm{PAH})$ e de creatinina foi considerado momento 1-M1 (controle). Os momentos 2 (M2), 3 (M3) e 4 (M4) foram realizados aos 30, 60 e 90 minutos após a injeção de solução fisiológica a 0,9\% - SF (Grupo 1) ou contraste radiológico (Grupo 2). Cada período de estudo durou 30 minutos. O estudo foi duplamente encoberto e os animais foram divididos em dois grupos experimentais, obedecendo ao critério de sorteio: Grupo 1 - G1 - controle,
SF, e Grupo 2 - G2 - contraste radiológico iônico de alta densidade (diatrizoato de meglumina e sódio). No início do momento 2 (M2), os animais receberam, por via intra-arterial, SF (Grupo 1) ou contraste radiológico (Grupo 2), ambos na dose de 1,24 mL. $\mathrm{kg}^{-1}$.

Em todos os animais foi realizada a seguinte seqüência experimental: jejum alimentar e restrição hídrica de 12 horas; pesagem; indução da anestesia; colocação e fixação do cão sobre a goteira de Claude Bernard; intubação traqueal; instalação da ventilação controlada com ar comprimido, empregando-se o aparelho de anestesia K. Takaoka, modelo 850-10; dissecção dos vasos sangüíneos; hidratação $(0,03$ $\mathrm{mL} \cdot \mathrm{kg}^{-1} \cdot \mathrm{min}^{-1}$ ); anestesia (infusão contínua para a manutenção); toracotomia no $4^{\circ}$ espaço intercostal para instalação da sonda fluxométrica na porção inicial da aorta ascendente, adaptação e calibração do fluxômetro eletromagnético (Blood Flowmeter) - Gould Statham, modelo SP 2202; injeção da dose inicial de PAH e creatinina (solução de PAH a 0,4\% e creatinina a $3 \%$ no volume de $1 \mathrm{~mL} . \mathrm{kg}^{-1}$ ) e a seguir infusão contínua de PAH a 1,6\% e creatinina a $4 \%$ em solução de glicose a $5 \%\left(0,015 \mathrm{~mL} \cdot \mathrm{kg}^{-1} \cdot \mathrm{min}^{-1}\right)$; todos os animais receberam dose complementar de cloreto de alcurônio $(0,06$ $\left.\mathrm{mg} \cdot \mathrm{kg}^{-1}\right)$. No final do experimento os cães foram sacrificados com injeção de cloreto de potássio. Foram estudados os seguintes atributos: pressão arterial média (PAM); freqüência cardíaca (FC); pressão da veia cava inferior ( $\mathrm{PVCl}$ ); fluxo sangüíneo renal (FSR); débito cardíaco (DC); hematócrito $(\mathrm{Ht})$; medidas da depuração de creatinina - ritmo de filtração glomerular (RFG) e do PAH - fluxo plasmático efetivo renal (FPER); resistência vascular renal (RVR); sódio, potássio e osmolalidade plasmática e urinária; temperatura retal $\left({ }^{\circ} \mathrm{C}\right)$. No final do experimento, foram retirados fragmentos do rim esquerdo, para exame histológico. Estes fragmentos foram colocados em solução de formol a 5\%; após fixação, foram incluídos em parafina e corados com hematoxilina-eosina. As lâminas foram examinadas por patologista que diagnosticou o tipo e a intensidade da lesão anátomo-patológica. Para cada variável foram calculadas a média ( $x$ ) e o desvio-padrão (s) em cada um dos momentos e foi utilizada a análise de variância, fatorial, inteiramente aleatória com testes de: interação entre grupo e momento, efeito de grupo e efeito de momento. Em todas as hipóteses testadas, o F calculado foi considerado significativo quando $p<0,05$. Os contrastes entre pares de médias foram analisados pelo teste de Tukey, com cálculo da diferença mínima significativa para alfa $=0,05$.

\section{RESULTADOS}

Os grupos foram homogêneos com relação ao peso e ao sexo.

Em ambos os grupos ocorreu aumento significativo da freqüência cardíaca (Tabela I), da depuração de sódio $(\mathrm{G} 1<\mathrm{G} 2$ em M3 e M4) (Tabela II) e da excreção urinária de sódio (G1 < G2 em M2, M3 e M4) (Tabela II). Também ocorreu em ambos os grupos diminuição significativa da osmolalidade urinária (Tabela II), do volume urinário e do potássio plasmático (G1 < G2 em M3 e M4) (Tabela II). 
O grupo controle (G1) apresentou aumento significativo do fluxo plasmático efetivo renal (Tabela I), do fluxo sangüíneo renal (Tabela I e Figura 4), da osmolalidade plasmática (Tabela II) e da diminuição significativa da depuração de potássio (Tabela II).

A infusão de contraste radiológico (G2) proporcionou aumento significativo do débito cardíaco, da resistência vascular renal (Tabela I), da depuração osmolar (G1 < G2 em M3 e M4) (Tabela II) e da excreção fracionária de sódio (G1 < G2 em M3 e M4) (Tabela II). Também proporcionou a diminuição do hematócrito (Tabela I), do ritmo de filtração glomerular (Tabela I), da fração de filtração (Tabela I) e da depuração de água livre (G1 < G2 em M3 e M4) (Tabela II).

Em ambos os grupos, de modo semelhante, foram encontrados animais com análise histológica normal ou com focos de infiltrado inflamatório mononuclear intersticial na cortical renal, ou, então, focos de necrose tubular evidenciados por áreas de cariólise.

Tabela I - Resultados da Freqüência Cardíaca, do Fluxo Plasmático Efetivo Renal, do Fluxo Sangüíneo Renal, do Débito Cardíaco, da Resistência Vascular Renal, do Hematócrito, do Ritmo de Filtração Glomerular e da Fração de Filtração

\begin{tabular}{|c|c|c|c|c|c|}
\hline \multirow[t]{2}{*}{ Atributos } & \multirow[t]{2}{*}{ Grupos } & \multicolumn{4}{|c|}{ Momentos } \\
\hline & & M1 & M2 & M3 & M4 \\
\hline \multirow[t]{2}{*}{ 1. Freqüência cardíaca - FC (bat.min $\left.{ }^{-1}\right)$} & G1 & $126 \pm 18$ & $137 \pm 10^{*}$ & $144 \pm 10^{*}$ & $143 \pm 13^{*}$ \\
\hline & G2 & $125 \pm 22$ & $132 \pm 22$ & $140 \pm 22^{*}$ & $141 \pm 17^{*}$ \\
\hline \multirow[t]{2}{*}{ 2. Fluxo plasmático efetivo renal - FPER $\left(\mathrm{mL} \cdot \mathrm{min}^{-1} \cdot \mathrm{kg}^{-1}\right)$} & G1 & $7,6 \pm 4,5$ & $11,5 \pm 5,2^{*}$ & $91 \pm 3,9$ & $7,2 \pm 3,7$ \\
\hline & G2 & $8,3 \pm 4,0$ & $99 \pm 5,3^{*}$ & $89 \pm 3,7$ & $6,4 \pm 5,5$ \\
\hline \multirow[t]{2}{*}{ 3. Fluxo sangüíneo renal - FSR (mL. $\left.\mathrm{min}^{-1} \cdot \mathrm{kg}^{-1}\right)$} & G1 & $12,3 \pm 7,3$ & $18,9 \pm 9,4^{*}$ & $15,2 \pm 7,4^{*}$ & $11,9 \pm 7,0$ \\
\hline & G2 & $14,6 \pm 7,6$ & $16,4 \pm 8,8$ & $16,2 \pm 7,1$ & $11,9 \pm 10,7$ \\
\hline \multirow[t]{2}{*}{ 4.Débito cardíaco - DC (L.min $\left.{ }^{-1}\right)$} & G1 & $0,86 \pm 0,49$ & $0,91 \pm 0,45$ & $0,83 \pm 0,47$ & $0,70 \pm 0,49$ \\
\hline & G2 & $1,19 \pm 0,69$ & $1,42 \pm 0,84^{*}$ & $1,26 \pm 0,73$ & $1,09 \pm 0,58$ \\
\hline \multirow[t]{2}{*}{ 5. Resistência vascular renal -RVR $\left(\mathrm{mmHg} \cdot \mathrm{mL}^{-1} \mathrm{~min}^{-1}\right)$} & G1 & $1,17 \pm 0,61$ & $0,80 \pm 0,55$ & $0,97 \pm 0,76$ & $1,10 \pm 0,57$ \\
\hline & G2 & $0,72 \pm 0,22$ & $0,65 \pm 0,23$ & $0,74 \pm 0,42$ & $1,28 \pm 0,78^{*}$ \\
\hline \multirow[t]{2}{*}{ 6. Hematocontraste radiológico $(\mathrm{Ht})-\%$} & $\mathrm{G}^{+}$ & $39 \pm 4$ & $37 \pm 5$ & $39 \pm 5$ & $38 \pm 5$ \\
\hline & G2 & $43 \pm 3$ & $40 \pm 3^{*}$ & $44 \pm 4$ & $45 \pm 4$ \\
\hline 7. Ritmo de filtração glomerular - RFG $\left(\mathrm{mL} \cdot \mathrm{min}^{-1} \cdot \mathrm{kg}^{-1}\right)$ & G1 & $3,44 \pm 0,92$ & $4,96 \pm 1,93$ & $4,13 \pm 1,47$ & $3,09 \pm 0,92^{*}$ \\
\hline \multirow[t]{2}{*}{ 8. Fração de filtração - FF } & G1 & $0,54 \pm 0,21$ & $0,48 \pm 0,18$ & $0,49 \pm 0,16$ & $0,46 \pm 0,11$ \\
\hline & G2 & $0,61 \pm 0,18$ & $0,47 \pm 0,12^{*}$ & $0,53 \pm 0,15^{*}$ & $0,59 \pm 0,13$ \\
\hline
\end{tabular}

${ }^{*} p<0,05 ;$ + diferença significativa em G1 e G2

Tabela II - Resultados da Depuração de Sódio, da Excreção Urinária de Sódio, da Osmolaridade Urinária, do Volume Urinário, do Potássio Plasmático, da Osmolalidade Plasmática, da Depuração de Potássio, da Depuração Osmolar, da Excreção Fracionária de Sódio e da Depuração de Água Livre

\begin{tabular}{|c|c|c|c|c|c|}
\hline \multirow[t]{2}{*}{ Atributos } & \multirow[t]{2}{*}{ Grupos } & \multicolumn{4}{|c|}{ Momentos } \\
\hline & & M1 & M2 & M3 & M4 \\
\hline \multirow[t]{2}{*}{ 1. Depuração de sódio - $\mathrm{D}_{\mathrm{Na}+}\left(\mathrm{mL} \cdot \mathrm{min}^{-1} \cdot \mathrm{kg}^{-1}\right)$} & $\mathrm{G} 1^{+}$ & $0,03 \pm 0,02$ & $0,06 \pm 0,05^{*}$ & $0,03 \pm 0,02$ & $0,02 \pm 0,01$ \\
\hline & G2 & $0,03 \pm 0,02$ & $0,09 \pm 0,05^{*}$ & $0,06 \pm 0,03$ & $0,04 \pm 0,02$ \\
\hline \multirow[t]{2}{*}{ 2. Excreção urinária de sódio $-\mathrm{EU}_{\mathrm{Na}+}\left(\mu \mathrm{Eq} \cdot \mathrm{min}^{-1}\right)$} & $\mathrm{G} 1^{+}$ & $45 \pm 31$ & $83 \pm 52^{*}$ & $35 \pm 15$ & $28 \pm 12$ \\
\hline & G2 & $46 \pm 21$ & $158 \pm 77^{*}$ & $107 \pm 60$ & $68 \pm 48$ \\
\hline \multirow[t]{2}{*}{ 3. Osmolalidade urinária - $\mathrm{U}_{\mathrm{osm}}\left(\mathrm{mOsm} . \mathrm{kgH}_{2} \mathrm{O}^{-1}\right)$} & G1 & $958 \pm 342$ & $784 \pm 273^{*}$ & $871 \pm 328^{*}$ & $945 \pm 378$ \\
\hline & G2 & $992 \pm 211$ & $592 \pm 155^{*}$ & $764 \pm 246$ & $897 \pm 335$ \\
\hline \multirow[t]{2}{*}{ 4. Volume urinário - VU $\left(\mathrm{mL} \cdot \mathrm{min}^{-1} \cdot \mathrm{kg}^{-1}\right)$} & $\mathrm{G} 1^{+}$ & $0,04 \pm 0,01$ & $0,07 \pm 0,05^{*}$ & $0,03 \pm 0,01$ & $0,02 \pm 0,01$ \\
\hline & G2 & $0,03 \pm 0,01$ & $0,12 \pm 0,05^{*}$ & $0,07 \pm 0,03^{*}$ & $0,04 \pm 0,02^{*}$ \\
\hline \multirow[t]{2}{*}{ 5. Potássio plasmático $-\mathrm{P}_{\mathrm{K}+}\left(\mathrm{mEq} \cdot \mathrm{L}^{-1}\right)$} & G1 & $3,6 \pm 0,5$ & $3,6 \pm 1,2$ & $3,0 \pm 0,2^{*}$ & $3,0 \pm 0,4^{*}$ \\
\hline & G2 & $3,5 \pm 0,5$ & $3,3 \pm 0,5$ & $3,0 \pm 0,3^{*}$ & $2,9 \pm 0,7^{*}$ \\
\hline \multirow[t]{2}{*}{ 6. Osmolalidade plasmática - $\mathrm{P}_{\mathrm{osm}}\left(\mathrm{mOsm} . \mathrm{kgH}_{2} \mathrm{O}^{-1}\right.$ ) } & G1 & $282 \pm 18$ & $281 \pm 23$ & $290 \pm 08^{*}$ & $292 \pm 06^{*}$ \\
\hline & G2 & $294 \pm 12$ & $299 \pm 09$ & $294 \pm 14$ & $298 \pm 10$ \\
\hline 7. Depuração de potássio $-\mathrm{D}_{\mathrm{K}+}\left(\mathrm{mL} \cdot \mathrm{min}^{-1} \cdot \mathrm{kg}^{-1}\right)$ & G1 & $0,87 \pm 0,41$ & $1,00 \pm 0,53$ & $0,62 \pm 0,24$ & $0,47 \pm 0,18^{*}$ \\
\hline \multirow[t]{2}{*}{ 8. Depuração osmolar - $\mathrm{D}_{\mathrm{osm}}\left(\mathrm{mL} \cdot \mathrm{min}^{-1} \cdot \mathrm{kg}^{-1}\right)$} & $\mathrm{G} 1^{+}$ & $0,12 \pm 0,04$ & $0,15 \pm 0,08^{*}$ & $0,08 \pm 0,03$ & $0,07 \pm 0,03$ \\
\hline & $\mathrm{G} 2^{*}$ & $0,08 \pm 0,03$ & $0,22 \pm 0,05^{*}$ & $0,16 \pm 0,05^{*}$ & $0,12 \pm 0,03$ \\
\hline \multirow[t]{2}{*}{ 9. Excreção fracionária de sódio - $E F_{\mathrm{Na}^{+}}(\%)$} & $\mathrm{G} 1^{+}$ & $0,93 \pm 0,43$ & $1,20 \pm 0,89^{*}$ & $0,61 \pm 0,23$ & $0,58 \pm 0,26$ \\
\hline & G2 & $0,55 \pm 0,30$ & $2,40 \pm 1,55^{*}$ & $1,56 \pm 0,97^{*}$ & $1,39 \pm 1,02$ \\
\hline \multirow[t]{2}{*}{ 10. Depuração de água livre - $\mathrm{D}_{\mathrm{H} 2 \mathrm{O}}\left(\mathrm{mL} \cdot \mathrm{min}^{-1}\right)$} & $\mathrm{G} 1^{+}$ & $-0,78 \pm 0,35$ & $-0,89 \pm 0,47^{*}$ & $-0,55 \pm 0,22$ & $-0,54 \pm 0,26$ \\
\hline & G2 & $-0,76 \pm 0,29$ & $1,25 \pm 0,49^{*}$ & $1,26 \pm 0,68^{*}$ & $-0,94 \pm 0,45$ \\
\hline
\end{tabular}

${ }^{*} p<0,05 ;+$ diferença significativa entre G1 e G2 


\section{DISCUSSÃO}

O tiopental sódico e o fentanil foram administrados em infusão contínua para proporcionar condições uniformes durante todo o período da experiência, evitando-se tanto o período de sobredose quanto os de concentrações abaixo dos níveis terapêuticos. Aosmolalidade urinária diminuiu em ambos os grupos, logo após a infusão de solução fisiológica e de contraste radiológico, mas seus valores continuaram elevados. Estes valores elevados da osmolalidade demonstraram que a restrição hídrica foi suficiente para causar a contração do volume extracelular.

Alguns estudos mostraram hipertensão temporária após injeção de contraste radiológico ${ }^{10-11}$. No presente estudo não houve alterações deste parâmetro, como em a outra pesqui$\mathrm{sa}^{12}$, mas existiu aumento da freqüência cardíaca e do débito cardíaco. Ocorreu diminuição temporária do hematócrito logo após a infusão de contraste radiológico. Este resultado também foi constatado por outros estudos ${ }^{9,13,14}$, e pode ser atribuído ao efeito osmótico da solução contida no contraste. Há relatos ${ }^{15,16}$ que comprovaram diminuição do FSR e do FPER em cães anestesiados com fentanil. Em ambos os grupos, houve elevação temporária do FPER e do FSR, sendo significativa apenas no grupo da solução fisiológica a 0,9\%. A possível explicação para os resultados deste estudo é a técnica anestésica empregada. Enquanto os trabalhos anteriores usaram a injeção em dose única, nesta pesquisa foi utilizada a infusão contínua do fentanil.

Foi demonstrada ${ }^{17}$ elevação temporária do FSR e do RFG após injeção de 2 ou $4 \mathrm{~mL} . \mathrm{kg}^{-1}$ de contraste. Após a injeção de $8 \mathrm{~mL} . \mathrm{kg}^{-1}$, não foram encontradas alterações do FSR, mas observou-se diminuição do RFG. No presente estudo, o RFG também diminuiu. Com relação ao FSR, pode ser atribuído o resultado diferente ao emprego de diferentes métodos nos dois estudos. Enquanto o primeiro estudo ${ }^{17}$ realizou a avaliação do FSR por meio do método fluxométrico, a presente pesquisa usou o método da depuração de PAH. Na técnica fluxométrica, o resultado obtido é imediato, enquanto que no método da depuração o resultado é a média do que aconteceu no período de 30 minutos. Neste último caso, resultados fugazes e de pouca significação clínica não são detectados. Concordante com esse estudo, outra pesquisa ${ }^{12}$ não encontrou alterações no FSR e verificou diminuição no RFG após a injeção de contraste radiológico. Nesse estudo, após a injeção de contraste, ocorreram diminuição do RFG e aumento da RVR, sendo significativos no último momento, demonstrando que a injeção de contraste causou somente alterações tardias.

AFF diminuiu temporariamente após a infusão de contraste radiológico e resultado similar foi obtido por Katzberg e col. ${ }^{18}$. Vários estudos também mostraram diminuição da depuração de creatinina após injeção de contraste radiológico de baixa ou alta osmolalidade ${ }^{3,19,20}$, ou também diminuição temporária deste parâmetro após contraste ${ }^{19,21}$. Alguns autores utilizaram a creatinina plasmática $\left(\mathrm{P}_{\mathrm{Cr}}\right)$ como parâmetro de estudo da função renal. Ocorreu aumento da $\mathrm{P}_{\mathrm{Cr}}$ quando foi administrado contraste radiológico simultaneamente com furosemida ${ }^{22,23}$ ou manitol ${ }^{23}$,em pacientes com $\mathrm{P}_{\mathrm{Cr}}$ basal normal ou elevada ${ }^{3} \mathrm{e}$ em pacientes com insuficiência renal ou com a associação desta e diabete melito ${ }^{24}$. Outro estudo mostrou que a $P_{C r}$ não se alterou significativamente com a administração de contraste radiológico ${ }^{25}$. Nesses estudos, a diferença pode ter sido a hidratação ou pode ter ocorrido redução no RFG, embora não tão acentuada a ponto de elevar a $\mathrm{P}_{\mathrm{Cr}}$.

Na literatura, há estudos correlacionando a resposta bifásica do FSR ao aumento da osmolalidade ${ }^{26,27}$.

Como outra hipótese para explicar os efeitos do contraste radiológico sobre a hemodinâmica renal, aventa-se a possibilidade da influência da solução hiperosmótica estimular o sistema renina-angiotensina ${ }^{28}$. Foi demonstrado ${ }^{29}$ que soluções hipertônicas injetadas na artéria renal de cães causaram rapidamente grande elevação da liberação de renina.

Logo após a injeção de contraste radiológico, o VU e a $D_{\text {osm }}$ aumentaram significativamente, sendo que $a D_{\text {osm }}$ retornou a valores basais no final do experimento. Também ocorreu diminuição temporária da $\mathrm{D}_{\mathrm{H} 2 \mathrm{O}}$.

Com relação ao volume urinário, a literatura é controversa pois há estudos ${ }^{19,30}$ mostrando sua diminuição após a injeção de contraste radiológico, enquanto outros ${ }^{10,12,13}$, semeIhante a este, mostraram aumento deste parâmetro. Também concordante com este estudo, outra pesquisa ${ }^{13}$ mostrou aumento da $\mathrm{D}_{\text {osm }}$ após injeção de contraste radiológico. Em ambos os grupos, ocorreu aumento da $\mathrm{D}_{\mathrm{Na}^{+}}, \mathrm{EU}_{\mathrm{Na}^{+}} \mathrm{eP}_{\mathrm{K}+} \mathrm{e}$ no grupo do contraste radiológico ocorreu também aumento da $E F_{\mathrm{Na}^{+}}$e diminuição do $U_{\mathrm{Na}^{+}} \mathrm{e} U_{\mathrm{K}+\text {. }}$ Estas últimas podem ser explicadas pela diluição induzida pela diurese osmótica. Alguns estudos, concordantes com este, mostraram aumento da $\mathrm{EU}_{\mathrm{Na}+}{ }^{12,31,32}$ e da $\mathrm{EF}_{\mathrm{Na}+}{ }^{32}$ após contraste radiológico iônico e não-iônico. Este fenômeno parece ser independente da osmolalidade do contraste. A excreção de sódio aumentada não pode ser explicada pelo conteúdo de sódio do contraste radiológico: o diatrizoato contém grande quantidade de sódio, enquanto no ioxilan, no iopamidol, no iohexol e em outros contrastes de baixa osmolalidade a concentração deste eletrólito é muito pequena. Uma possível explicação para o aumento da excreção urinária de sódio pode ser a diurese osmótica provocada por todos os tipos de contraste radiológico, independentemente do conteúdo de sódio e do contraste radiológico ser ou não hiperosmótico.

Aeliminação do potássio é realizada, principalmente, pelos rins e a taxa é diretamente proporcional à sobrecarga deste íon no organismo. Também, quando a quantidade de sódio está muito alta na luz tubular, ocorre entrada de sódio para a célula tubular e estimulação da bomba de sódio, com entrada de potássio na célula e posterior eliminação na luz tubular ${ }^{33}$. Isto pode explicar a diminuição de potássio plasmático nos dois últimos momentos, em ambos os grupos deste experimento.

Com relação ao exame anatomopatológico, não houve diferença entre grupos. Os animais de ambos os grupos apresentaram análise histológica normal ou infiltrado inflamatório intersticial de pequena a grande intensidade ou focos de necrose tubular. 
Um dos primeiros trabalhos realizados em nosso Departamento ${ }^{34}$ encontrou, na maioria dos cães, alterações histológicas compatíveis com o quadro de pielonefrite crônica. O autor concluiu que, em cães, é muito comum o diagnóstico anatomopatológico de pielonefrite crônica. O resultado semelhante anatomopatológico obtido em ambos os grupos demonstra que não houve lesões renais causadas pelo contraste radiológico.

Assim, nas condições deste estudo, a injeção intra-arterial de contraste radiológico em animais com redução do volume extracelular causou efeito bifásico na função renal. Inicialmente provocou aumento da diurese e da excreção de sódio, mas, posteriormente, houve piora das condições hemodinâmicas e, conseqüentemente, da função renal, com aumento da resistência vascular renal e diminuição do ritmo de filtração glomerular.

\section{Cardiovascular and Renal Effects of Intra-Arterial Injection of Ionic Radiological Contrast in Dogs under Fluid Restriction}

Marisa Aparecida Lima Verderese, M.D.; Pedro Thadeu Galvão Vianna TSA, M.D.; Yara Marcondes Machado Castiglia TSA, M.D.; Luiz Antonio Vane TSA, M.D.

\section{INTRODUCTION}

Radiological contrast administration is still one of the most common iatrogenic causes of acute renal failure acquired in the hospital ${ }^{1-4}$. Although the pathogenesis of such condition is still not totally understood, it seems to be due to medullary ischemia caused by decreased renal blood flow ${ }^{1-4}$.

Intravenous fluids, mannitol and furosemide, have been recommended to prevent radiological contrast-induced nephropathy, but the efficacy them has not yet been proven ${ }^{5}$. Recently, intravenous acetylcysteine $\left(150 \mathrm{mg} \cdot \mathrm{kg}^{-1}\right) 30 \mathrm{~min}-$ utes before radiological contrast administration has been indicated, followed by $50 \mathrm{mg} \cdot \mathrm{kg}^{-1}$ four hours after radiological procedure. Both acethylcystein doses were diluted in $500 \mathrm{~mL}$ saline. Results have shown renal protection against nephrotoxicity caused by radiological contrast.

The incidence of nephropathy by radiological contrast may reach much higher values In the presence of predisposing factors, among them: dehydration, diabetes mellitus, multiple mieloma, advanced age, heart disease, use of diuretics, renal failure and exams with radiological contrast performed in short time periods ${ }^{2,8,9}$.

Radiological contrast during anesthesia may be intra-arterially injected for diagnostic purposes. So, the understanding of acute and immediate effects of radiological contrast on major renal functions is critical.
This study aimed at evaluating acute effects of radiological contrast in fluid restriction states. For this purpose, the experiment was performed with dogs under fluid restriction to evaluate immediate renal and cardiovascular effects after intra-arterial injection of high osmolarity radiological contrast.

\section{METHODS}

After the Animal Experiment Ethics Committee, Faculdade de Medicina, Botucatu, UNESP approval, 16 male, adult, mixed-breed dogs weighing 7 to $17 \mathrm{~kg}$ and supplied by the Central Lab Animals Facility, UNESP, were involved in this study. Anesthesia was induced with sodium thiopental (15 $\left.\mathrm{mg} \cdot \mathrm{kg}^{-1}\right)$, fentanyl $\left(15 \mu \mathrm{g} \cdot \mathrm{kg}^{-1}\right)$, and alcuronium $\left(0.2 \mathrm{mg} \cdot \mathrm{kg}^{-1}\right)$, followed by $40 \mu \mathrm{g} \cdot \mathrm{kg}^{-1} \cdot \mathrm{min}^{-1}$ sodium thiopental and 0.1 $\mu \mathrm{g} . \mathrm{kg}^{-1} \mathrm{~min}^{-1}$ fentanyl in continuous infusion throughout the experiment. Moment 1 (control) was defined as 30 minutes after sodium para-aminohipurate $(\mathrm{PAH})$ and creatinine infusion. Moments 2 (M2), 3 (M3) and 4 (M4) were defined as 30 , 60 and 90 minutes after $0.9 \%$ saline (Group 1 ) or radiological contrast injection (Group G 2). Each study period lasted 30 minutes.

This was a double-blind study and animals were randomly distributed in two experimental groups: Group 1 - G1 - control, SS, and Group 2 - G2 - high intensity ionic radiological contrast (meglumine diatrizoate and sodium). To start moment 2 (M2) animals received $1.24 \mathrm{~mL} \cdot \mathrm{kg}^{-1}$ intra-arterial saline (Group 1) or radiological contrast (Group 2).

The following experimental sequence was performed for all animals: feed and fluid restriction for 12 hours; weighing; anesthetic induction; dog placement and fixation on Claude Bernard device; tracheal intubation; controlled ventilation with compressed air, using K. Takaoka model 850-10 anesthesia machine; blood vessels dissection; hydration $(0.03$ $\mathrm{mL} . \mathrm{kg}^{-1} \cdot \mathrm{min}^{-1}$ ); anesthesia (continuous infusion for maintenance); thoracotomy in the $4^{\text {th }}$ intercostal space to place the flowmetric probe in the initial portion of ascending aorta, adaptation and calibration of electromagnetic flowmeter (Blood Flowmeter) - Gould Statham model SP 2202; injection of initial PAH and creatinine dose $\left(1 \mathrm{~mL} . \mathrm{kg}^{-1}\right.$ of $0.4 \% \mathrm{PAH}$ and $3 \%$ creatinine) followed by $1.6 \% \mathrm{PAH}$ and $4 \%$ creatinine continuous infusion in $5 \%$ glucose solution $\left(0.015 \mathrm{~mL} \cdot \mathrm{kg}^{-1} \cdot \mathrm{min}^{-1}\right)$; all animals received supplemental alcuronium $\left(0.06 \mathrm{mg} \cdot \mathrm{kg}^{-1}\right)$. At experiment end animals were sacrificed with potassium chloride. The following attributes were evaluated: mean blood pressure (MBP); heart rate (HR); inferior vena cava pressure (IVCP); renal blood flow (RBF); cardiac output $(\mathrm{CO})$; hematocrit $(\mathrm{Ht})$; creatinine clearance - glomerular and $\mathrm{PAH}$ filtration rate - effective renal plasma flow (ERPF); renal vascular resistance (RVR); sodium, potassium, and plasma and urinary osmolality; rectal temperature $\left({ }^{\circ} \mathrm{C}\right)$.

At experiment end, fragments of the left kidney were removed for histological exam. These fragments were formalin fixed processed for inclusion in paraffin and stained with hematoxylin-eosin. Sections were examined by the pathologist who diagnosed type and intensity of pathological injury. 
Mean (x) and standard deviation (s) were calculated for each variable in each moment and fully randomized factorial analysis of variance was used with the following tests: interaction between group and moment, group effect and moment effect. In all tested hypothesis, $F$ were considered significant when $p$ $<0.05$. Contrasts between pairs of means were analyzed by Tukey's test, with calculation of minimum significant difference for alpha $=0.05$

\section{RESULTS}

Groups were homogeneous in weight and gender.

There has been significant increase in heart rate (Table I), sodium clearance ( $\mathrm{G} 1<\mathrm{G} 2$ in M3 and M4) (Table II) and sodium urinary excretion ( $\mathrm{G} 1<\mathrm{G} 2$ in M2, M3 and M4) (Table II) for both groups. There has also been significant decrease in uri-

Table I - Results of Heart Rate, Effective Renal Plasma Flow, Renal Blood Flow, Cardiac Output, Renal Vascular Resistance, Hematocrit, Glomerular Filtration Rate and Filtration Fraction

\begin{tabular}{|c|c|c|c|c|c|}
\hline \multirow[t]{2}{*}{ Attributes } & \multirow[t]{2}{*}{ Groups } & \multicolumn{4}{|c|}{ Moments } \\
\hline & & M1 & $\mathrm{M} 2$ & M3 & M4 \\
\hline \multirow[t]{2}{*}{ 1. Heart rate - HR (beat.min ${ }^{-1}$ ) } & G1 & $126 \pm 18$ & $137 \pm 10^{*}$ & $144 \pm 10^{*}$ & $143 \pm 13^{*}$ \\
\hline & G2 & $125 \pm 22$ & $132 \pm 22$ & $140 \pm 22^{*}$ & $141 \pm 17^{*}$ \\
\hline \multirow[t]{2}{*}{ 2. Effective renal plasma flow - ERPF $\left(\mathrm{mL} \cdot \mathrm{min}^{-1} \cdot \mathrm{kg}^{-1}\right)$} & G1 & $7.6 \pm 4.5$ & $11.5 \pm 5.2^{*}$ & $91 \pm 3.9$ & $7.2 \pm 3.7$ \\
\hline & G2 & $8.3 \pm 4.0$ & $99 \pm 5.3^{*}$ & $89 \pm 3.7$ & $6.4 \pm 5.5$ \\
\hline \multirow[t]{2}{*}{ 3. Renal blood flow - RBF (mL. $\left.\min _{-1} \cdot \mathrm{kg}_{-1}\right)$} & G1 & $12.3 \pm 7.3$ & $18.9 \pm 9.4^{*}$ & $15.2 \pm 7.4^{*}$ & $11.9 \pm 7.0$ \\
\hline & G2 & $14.6 \pm 7.6$ & $16.4 \pm 8.8$ & $16.2 \pm 7.1$ & $11.9 \pm 10.7$ \\
\hline \multirow[t]{2}{*}{ 4.Cardiac output - CO (L. $\left.\min _{-1}\right)$} & G1 & $0.86 \pm 0.49$ & $0.91 \pm 0.45$ & $0.83 \pm 0.47$ & $0.70 \pm 0.49$ \\
\hline & G2 & $1.19 \pm 0.69$ & $1.42 \pm 0.84^{*}$ & $1.26 \pm 0.73$ & $1.09 \pm 0.58$ \\
\hline \multirow[t]{2}{*}{ 5. Renal vascular resistance - RVR $\left(\mathrm{mmHg} \cdot \mathrm{mL}^{-1} \cdot \mathrm{min}^{-1}\right)$} & G1 & $1.17 \pm 0.61$ & $0.80 \pm 0.55$ & $0.97 \pm 0.76$ & $1.10 \pm 0.57$ \\
\hline & G2 & $0.72 \pm 0.22$ & $0.65 \pm 0.23$ & $0.74 \pm 0.42$ & $1.28 \pm 0.78^{*}$ \\
\hline \multirow[t]{2}{*}{ 6. Hematocrit $(\mathrm{Ht})-\%$} & $\mathrm{G} 1^{+}$ & $39 \pm 4$ & $37 \pm 5$ & $39 \pm 5$ & $38 \pm 5$ \\
\hline & G2 & $43 \pm 3$ & $40 \pm 3^{*}$ & $44 \pm 4$ & $45 \pm 4$ \\
\hline 7. Glomerular filtration rate - GFR $\left(\mathrm{mL} \cdot \mathrm{min}^{-1} \cdot \mathrm{kg}^{-1}\right)$ & G1 & $3.44 \pm 0.92$ & $4.96 \pm 1.93$ & $4.13 \pm 1.47$ & $3.09 \pm 0.92^{*}$ \\
\hline \multirow[t]{2}{*}{ 8. Filtration fraction - FF } & G1 & $0.54 \pm 0.21$ & $0.48 \pm 0.18$ & $0.49 \pm 0.16$ & $0.46 \pm 0.11$ \\
\hline & G2 & $0.61 \pm 0.18$ & $0.47 \pm 0.12^{*}$ & $0.53 \pm 0.15^{*}$ & $0.59 \pm 0.13$ \\
\hline
\end{tabular}

* $p<0.05 ;+$ significant difference in $\mathrm{G} 1$ and $\mathrm{G} 2$

Table II - Results of Sodium Clearance, Sodium Urinary Excretion, Urinary Osmolarity, Urinary Volume, Plasma Potassium, Plasma Osmolality, Potassium Clearance, Osmolar Clearance, Sodium Fractional Excretion and Free Water Clearance

\begin{tabular}{|c|c|c|c|c|c|}
\hline \multirow[t]{2}{*}{ Attributes } & \multirow[t]{2}{*}{ Groups } & \multicolumn{4}{|c|}{ Moments } \\
\hline & & M1 & M2 & M3 & M4 \\
\hline \multirow[t]{2}{*}{ 1. Sodium clearance $-\mathrm{C}_{\mathrm{Na}+}\left(\mathrm{mL} \cdot \mathrm{min}^{-1} \cdot \mathrm{kg}^{-1}\right)$} & $\mathrm{G} 1^{+}$ & $0.03 \pm 0.02$ & $0.06 \pm 0.05^{*}$ & $0.03 \pm 0.02$ & $0.02 \pm 0.01$ \\
\hline & G2 & $0.03 \pm 0.02$ & $0.09 \pm 0.05^{\star}$ & $0.06 \pm 0.03$ & $0.04 \pm 0.02$ \\
\hline \multirow[t]{2}{*}{ 2. Sodium urinary excretion - $\mathrm{UE}_{\mathrm{Na}+}\left(\mu \mathrm{Eq} \cdot \mathrm{min}^{-1}\right)$} & $\mathrm{G} 1^{+}$ & $45 \pm 31$ & $83 \pm 52^{*}$ & $35 \pm 15$ & $28 \pm 12$ \\
\hline & G2 & $46 \pm 21$ & $158 \pm 77^{*}$ & $107 \pm 60$ & $68 \pm 48$ \\
\hline \multirow[t]{2}{*}{ 3. Urinary osmolality - $\mathrm{U}_{\mathrm{osm}}\left(\mathrm{mOsm} . \mathrm{kgH}_{2} \mathrm{O}^{-1}\right)$} & G1 & $958 \pm 342$ & $784 \pm 273^{*}$ & $871 \pm 328^{*}$ & $945 \pm 378$ \\
\hline & G2 & $992 \pm 211$ & $592 \pm 155^{*}$ & $764 \pm 246$ & $897 \pm 335$ \\
\hline \multirow[t]{2}{*}{ 4. Urinary volume - UV (mL. $\left.\mathrm{min}^{-1} \cdot \mathrm{kg}^{-1}\right)$} & $\mathrm{G} 1^{+}$ & $0.04 \pm 0.01$ & $0.07 \pm 0.05^{*}$ & $0.03 \pm 0.01$ & $0.02 \pm 0.01$ \\
\hline & G2 & $0.03 \pm 0.01$ & $0.12 \pm 0.05^{*}$ & $0.07 \pm 0.03^{*}$ & $0.04 \pm 0.02 *$ \\
\hline \multirow[t]{2}{*}{ 5. Plasma potassium - $\mathrm{P}_{\mathrm{K}+}\left(\mathrm{mEq} \cdot \mathrm{L}^{-1}\right)$} & G1 & $3.6 \pm 0.5$ & $3.6 \pm 1.2$ & $3.0 \pm 0.2^{*}$ & $3.0 \pm 0.4^{*}$ \\
\hline & G2 & $3.5 \pm 0.5$ & $3.3 \pm 0.5$ & $3.0 \pm 0.3^{*}$ & $2.9 \pm 0.7^{*}$ \\
\hline \multirow{2}{*}{ 6. Plasma osmolality - $\mathrm{P}_{\mathrm{osm}}\left(\mathrm{mOsm} . \mathrm{kgH}_{2} \mathrm{O}^{-1}\right)$} & G1 & $282 \pm 18$ & $281 \pm 23$ & $290 \pm 08^{*}$ & $292 \pm 06^{*}$ \\
\hline & G2 & $294 \pm 12$ & $299 \pm 09$ & $294 \pm 14$ & $298 \pm 10$ \\
\hline 7. Potassium clearance $-\mathrm{C}_{\mathrm{K}+}\left(\mathrm{mL} \cdot \mathrm{min}^{-1} \cdot \mathrm{kg}^{-1}\right)$ & G1 & $0.87 \pm 0.41$ & $1.00 \pm 0.53$ & $0.62 \pm 0.24$ & $0.47 \pm 0.18^{*}$ \\
\hline \multirow[t]{2}{*}{ 8. Osmolar clearance $-\mathrm{C}_{\mathrm{osm}}\left(\mathrm{mL} \cdot \mathrm{min}^{-1} \cdot \mathrm{kg}^{-1}\right)$} & $\mathrm{G}^{+}$ & $0.12 \pm 0.04$ & $0.15 \pm 0.08^{*}$ & $0.08 \pm 0.03$ & $0.07 \pm 0.03$ \\
\hline & $\mathrm{G} 2^{*}$ & $0.08 \pm 0.03$ & $0.22 \pm 0.05^{\star}$ & $0.16 \pm 0.05^{*}$ & $0.12 \pm 0.03$ \\
\hline \multirow[t]{2}{*}{ 9. Sodium fractional excretion - $\mathrm{FE}_{\mathrm{Na}+}(\%)$} & $\mathrm{G} 1^{+}$ & $0.93 \pm 0.43$ & $1.20 \pm 0,89^{*}$ & $0,61 \pm 0,23$ & $0,58 \pm 0,26$ \\
\hline & G2 & $0,55 \pm 0,30$ & $2,40 \pm 1,55^{*}$ & $1,56 \pm 0,97^{*}$ & $1,39 \pm 1,02$ \\
\hline \multirow[t]{2}{*}{ 10. Free water clearance $-\mathrm{C}_{\mathrm{H} 2 \mathrm{O}}\left(\mathrm{mL} \cdot \mathrm{min}^{-1}\right)$} & $\mathrm{G} 1^{+}$ & $-0.78 \pm 0.35$ & $-0.89 \pm 0.47^{*}$ & $-0.55 \pm 0.22$ & $-0.54 \pm 0.26$ \\
\hline & $\mathrm{G} 2$ & $-0.76 \pm 0.29$ & $1.25 \pm 0.49^{*}$ & $1.26 \pm 0.68^{*}$ & $-0.94 \pm 0.45$ \\
\hline
\end{tabular}

* $p<0.05 ;+$ significant difference between G1 and G2 
nary osmolality (Table II), urinary volume and plasma potassium (G1 < G2 in M3 and M4) (Table II) for both groups. Control group (G1) had significant increase in effective renal plasma flow (Table I), renal blood flow (Table I and Figure 4) and plasma osmolality (Table II), and significant decrease in potassium clearance (Table II).

Radiological contrast infusion (G2) has promoted significant increase in cardiac output, renal vascular resistance (Table I), osmolar clearance (G1 < G2 in M3 and M4) (Table II) and sodium fractional excretion ( $\mathrm{G} 1<\mathrm{G} 2$ in M3 and M4) (Table II). It has also promoted decrease in hematocrit (Table I), glomerular filtration rate (Table I), filtration fraction (Table I) and free water clearance (G1 < G2 in M3 and M4) (Table II). In both groups there were animals with normal histological analysis or with interstitial mononuclear inflammatory infiltrate in renal cortical, or else, tubular necrosis focuses evidenced by karyolysis areas.

\section{DISCUSSION}

Sodium thiopental and fentanyl were administered in continuous infusion to provide uniform conditions throughout the experiment, preventing both overdose and concentrations below therapeutic levels. Urinary osmolality has decreased for both groups soon after saline or radiological contrast infusion, but its values remained high. These high osmolality values have shown that fluid restriction was enough to promote extracellular volume contraction.

Some studies have shown temporary hypertension after radiological contrast injection ${ }^{10-11}$. There have been no changes in this parameter in our study, similar to another study ${ }^{12}$, but there was increased heart rate and urinary output. There has been temporary hematocrit decrease after radiological contrast infusion. This result has been also observed by different studies ${ }^{9,13,14}$, and may be attributed to the osmotic effect of the solution in the contrast.

There are reports ${ }^{15,16}$ proving RBF and ERPF increase in dogs anesthetized with fentanyl. In both groups, there has been temporary RBF and ERPF increase which was however significant only for the $0.9 \%$ saline group. Possible explanation for our results would be the anesthetic technique. While previous studies have used bolus injection, we have used fentanyl in continuous infusion.

Temporary increase in RBF and GFR after 2 or $4 \mathrm{~mL}^{\mathrm{kg}} \mathrm{kg}^{-1}$ contrast have been shown ${ }^{17}$. After $8 \mathrm{~mL} . \mathrm{kg}^{-1}$, no changes in RBF were found, but there has been decrease in GFR. In our study, GFR has also decreased. As to RBF, different results may be attributed to different methods used by both studies. While the first study ${ }^{17}$ evaluated RBF by flowmetric method, our research has used PAH clearance method. In the flowmetric technique, results is immediate, while with the clearance method, result is the mean of what has happened during 30 minutes.

In this latter method, fugacious results with low clinical significance are not detected. In agreement with this study, a different study ${ }^{12}$ has not found RBF changes but has observed GFR decrease after radiological contrast injection. In our study, there have been RBF decrease and RVR increase after contrast injection, being significant in the final moment and showing that contrast injection has promoted only late changes.

FF has temporarily decreased after radiological contrast and similar result was obtained by Katzberg et al. ${ }^{18}$. Several studies have also shown decreased creatinine clearance after low or high osmolality radiological contrast ${ }^{3,19,20}$, or also temporary decrease of this parameter after contrast ${ }^{19,21}$. Some authors have used plasma creatinine $\left(\mathrm{P}_{\mathrm{Cr}}\right)$ as parameter for evaluating renal function. There has been $\mathrm{P}_{\mathrm{Cr}}$ increase when radiological contrast was simultaneously administered with furosemide ${ }^{22,23}$ or mannitol ${ }^{23}$, in patients with normal or increased baseline $\mathrm{P}_{\mathrm{Cr}}{ }^{3}$ and in patients with renal failure alone or associated to diabetes mellitus ${ }^{24}$. A different study has shown that $\mathrm{P}_{C r}$ was not significantly changed after radiological contrast ${ }^{25}$. The difference in those studies might have been hydration, of there might have been GFR decrease although not so significant to increase $\mathrm{P}_{\mathrm{Cr}}$.

Another hypothesis to explain radiological contrast effects on renal hemodynamics would be the possibility of hyperosmotic solutions stimulating rennin-angiotensin system ${ }^{28}$. It has been shown ${ }^{29}$ that hypertonic solutions injected in the renal artery of dogs would promptly promote major increase in rennin release.

UV and $\mathrm{C}_{\text {osm }}$ have significantly increased soon after radiological contrast injection, but $\mathrm{C}_{\text {osm }}$ has returned to baseline levels at the end of the experiment. There has also been temporary $\mathrm{C}_{\mathrm{H} 2 \mathrm{O}}$ decrease.

Literature is controversial with regard to urinary volume since there are studies ${ }^{19,30}$ showing its decrease after radiological contrast, while others ${ }^{10,12,13}$ have shown increase in this parameter, similarly to our study. Also in line with our study, a different research ${ }^{13}$ has shown increased $C_{\text {osm }}$ after radiological contrast injection.

Both groups have shown increased $\mathrm{C}_{\mathrm{Na}^{+}}, \mathrm{UE}_{\mathrm{Na}^{+}}$e $\mathrm{P}_{\mathrm{K}+}$ while in the radiological contrast group there has also been increased $\mathrm{FE}_{\mathrm{Na}+}$ and decreased $\mathrm{U}_{\mathrm{Na}+}$ and $\mathrm{U}_{\mathrm{K}+\text {. }}$. The two latter could be explained by osmotic diuresis-induced dilution. Some studies, in agreement with ours, have shown increased $U_{\mathrm{Na}^{+}}{ }^{12,31,32}$ and $\mathrm{FE}_{\mathrm{Na}+}{ }^{32}$ after ionic and non-ionic radiological contrast, which seems to be independent of contrast osmolality. Increased sodium excretion cannot be explained by sodium content in radiological contrast: diatrizoate has high sodium concentration, while ioxilan, iopamidol, iohexol and other low osmolality contrasts have very low concentrations of this electrolyte. A possible explanation for increased sodium urinary excretion could be osmotic diuresis promoted by all radiological contrasts, regardless of sodium content and of radiological contrast being or not hyperosmotic.

Potassium is primarily excreted by kidneys and the rate is a direct function of this ion overload in the body. Also, when sodium is too high in the tubular lumen, there is sodium entrance in the tubular cell and sodium pump stimulation with potassium entering the cell and then being excreted in the tubular lumen ${ }^{33}$. This may explain decreased plasma potassium in the two final moments in both groups in our experiment. 
There has been no difference between groups in pathological findings. Both groups presented normal histological analysis or interstitial inflammatory infiltrate of mild to severe intensity or tubular necrosis focuses.

One of the first studies performed in our Department ${ }^{34}$ has found, in most dogs, histological changes compatible with chronic pyelonephritis. The author has concluded that, in dogs, chronic pyelonephritis is a frequent pathological diagnosis. Similar pathological result obtained for both groups has shown that there were no radiological contrast-induced renal injuries.

So, in the conditions of this study, intra-arterial radiological contrast in animals with decreased extracellular volume has promoted a two-phase effect on renal function. Initially it promoted increased diuresis and sodium excretion, but then the hemodynamic conditions impaired and, as a consequence, renal function impaired, with increased renal vascular resistance and decreased glomerular filtration rate.

\section{REFERÊNCIAS - REFERENCES}

01. Hou SH, Bushinsky DA, Wish JB et al - Hospital-acquired renal insufficiency: a prospective study. Am J Med, 1983;74:243-248.

02. Solomon R - Radiocontrast-induced nephropathy. Semin Nephrol, 1998;18:551-557.

03. Gupta RK, Kapoor A, Tewari S et al - Captopril for prevention of contrast-induced nephropathy in diabetic patients: a randomized study. Indian Heart J, 1999;51:521-526.

04. Shammas NW, Kapalis MJ, Harris M et al - Aminophylline does not protect against radiocontrast nephropathy in patients undergoing percutaneous angiographic procedures. J Invasive Cardiol, 2001;13:738-740.

05. Berns AS - Nephrotoxicity of contrast media. Kidney Int, 1989;36:730-740.

06. Birck R, Krzossok S, Markowetz F et al - Acetylcysteine for prevention of contrast nephropathy: meta-analysis. Lancet, 2003:362;598-603

07. Baker CS, Wragg A, Kumar S et al - A rapid protocol for the prevention of contrast-induced renal dysfunction: the RAPPID study. J Am Coll Cardiol, 2003:41;2114-2118

08. Morcos SK, Thomsen HS, Webb JA - Contrast-media-induced nephrotoxicity: a consensus report. Contrast Media Safety Committee, European Society of Urogenital Radiology (ESUR). Eur Radiol, 1999;9:1602-1613.

09. Lepor NE, Mathur VS - Radiocontrast nephropathy. Curr Interv Cardiol Rep, 2000;2:335-341.

10. Nygren A, Ulfendahl HR - Effects of high- and low-osmolar contrast media on renal plasma flow and glomerular filtration rate in euvolaemic and dehydrated rats. A comparison between ioxithalamate, iopamidol, iohexol and ioxaglate. Acta Radiol, 1989;30:383-389.

11. Sunnegardh O, Hietala SO, Holtz E - Systemic, pulmonary and renal haemodinamic and renal morphologic effects of intravenously infused iodixanol. A study in the pig of a new iso-osmolar contrast medium. Acta Radiol, 1990;31:513-518.

12. Brooks DP, DePalma PD - Blockade of radiocontrast-induced nephrotoxicity by the endothelin receptor antagonist, SB 209670. Nephron, 1996;72:629-636.

13. Jakobsen JA, Berg KJ, Waaler A et al - Renal effects of the non-ionic contrast medium tiopental after intravenous injection in healthy volunteers. Acta Radiol, 1990;31 87-91.
14. Buyan N, Arab M, Hasanoglu E et al - The effects of contrast media on renal function in children: comparison of ionic and non-ionic agents. Turk J Pediatr, 1995;37:305-313.

15. Braz JRC, Vianna PTG, Hossne WS - Efeitos do fentanil, droperidol e inoval sobre a função renal e eletrólitos (sódio e potássio): estudo experimental no cão. Rev Bras Anestesiol, 1976;26:56-73

16. Castiglia YMM, Braz JRC, Vianna PTG et al - Effects of high-dose fentanyl on renal function in dogs. São Paulo Med J, 1997;115:1433-1439.

17. Forrest JB, Howards SS, Gillenwater JY - Osmotic effects of intravenous contrast agents on renal function. J Urol, 1981;125:147-150.

18. Katzberg RW, Morris TW, Schulman G et al - Reactions to intravenous contrast media. Part II: Acute renal response in euvolemic and desidrated dogs. Radiology, 1983;147:331-334.

19. Katholi RE, Taylor GJ, Woods WT et al - Nephrotoxicity of nonionic low-osmolality versus ionic high-osmolality contrast media: a prospective double-blind randomized comparison in human beings. Radiology, 1993;186:183-187.

20. Jakobsen JA, Berg KJ, Brodahl U et al - Renal effects of nonionic contrast media after cardioangiography. Acta Radiol, 1994;35:191-196.

21. Laranja SM, Ajzen H, Schor $\mathrm{N}$ - Nephrotoxicity of low-osmolality contrast media. Ren Fail, 1997;19:307-314.

22. Weinstein JM, Heyman S, Brezis M - Potential deleterious effect of furosemide in radiocontrast nephropathy. Nephron, 1992;62:413-415.

23. Solomon R, Werner C, Mann D et al - Effects of saline, mannitol, and furosemide to prevent acute decreases in renal function induced by radiocontrast agents. N Engl J Med, 1994;331:1416-1420.

24. Apelqvist J, Torffvit O, Agardh CD - The effect of the non-ionic contrast medium iohexol on glomerular and tubular function in diabetic patients. Diabet Med, 1996;13:487-492.

25. Stevens MA, McCullough PA, Tobin KJ et al - A prospective randomized trial of prevention measures in patients at high risk for contrast nephropathy: results of the P.R.I.N.C.E study. J Am Coll Cardiol, 1999;33:403-411.

26. Chou CC, Hook JB, Hsieh CP et al - Effects of radiopaque dyes on renal vascular resistance. J Lab Clin Med, 1971;78:705-712.

27. Katzberg RW, Morris TW, Burgener FA et al - Renal renin and hemodynamic responses to selective renal artery catheterization and angiography. Invest Radiol, 1977;12:381-388.

28. Caldicott WJ, Hollenberg NK, Abrams HL - Characteristics of response of renal vascular bed to contrast media. Evidence for vasoconstriction induced by renin-angiotensin system. Invest Radiol, 1970;5:539-547.

29. Young DB, Rostorfer HH - Renin release responses to acute alterations in renal arterial osmolarity. Am J Physiol, 1973;225:1009-1014.

30. Spangberg-Viklund B, Nikonoff T, Lundberg M et al - Acute renal failure caused by low-osmolar radiographic contrast media in patients with diabetic nephropathy. Scand J Urol Nephrol, 1989;23:315-317.

31. Garibotto G, Saffioti S, Garlaschi G et al - Comparative effects of nonionic (iopamidol) and ionic (sodium and meglumine diatrizoate) contrast media for urography on urinary excretion of water solutes. Urol Radiol, 1986;8:199-203.

32. Haller C, Meyer M, Scheele T et al - Radiocontrast-induced natriuresis associated with increased urinary urodilatin excretion. J Intern Med, 1998;243:155-162.

33. Malnic G, Marcondes M - Fisiologia Renal. $3^{\text {a }}$ Ed, São Paulo: Pedagógica e Universitária, 1986;236. 
34. Vianna PTG - Efeitos da cetamina sobre a função renal e eletrólitos (sódio e potássio): estudo experimental no cão. Rev Bras Anestesiol, 1974;24:503-517.

\section{RESUMEN}

Verderese MAL, Vianna PTG, Castiglia YMM, Vane LA - Efectos Cardiovasculares y Renales de la Inyección Intra-Arterial de Contraste Radiológico lónico en Perros con Restricción Hídrica

JUSTIFICATIVA Y OBJETIVOS: El objetivo de esta pesquisa fue estudiar los efectos agudos del contraste radiológico en situaciones de restricción de volumen, evaluándose los efectos renales y cardiovasculares después de inyección intra-arterial de contraste radiológico de alta osmolaridad.

MÉTODO: Participaron del estudio 16 perros anestesiadas con tiopental sódico $\left(15 \mathrm{mg} \cdot \mathrm{kg}^{-1}\right)$ y fentanil $\left(15 \mu \mathrm{g} \cdot \mathrm{kg}^{-1}\right)$ en bolus, seguido de infusión continuada en las dosis de $40 \mu \mathrm{g} \cdot \mathrm{kg}^{-1} \cdot \mathrm{min}^{-1}$ (tiopental sódico) y $0,1 \mu \mathrm{g} \cdot \mathrm{kg}^{-1} \cdot \mathrm{min}^{-1}$ (fentanil). Fue hecha hidratación con solución de glucosa a $5 \%\left(0,03 \mathrm{~mL} \cdot \mathrm{kg}^{-1} \cdot \mathrm{min}^{-1}\right)$ y la ventilación pulmonar fue controlada mecánicamente con aire comprimido. Fueron verificados los siguientes atributos: frecuencia cardiaca (FC); presión arterial media (PAM); presión de la vena cava inferior (PVI); débito cardíaco (DC); hematocrito $(\mathrm{Ht})$; flujo plasmático efectivo renal (FPER); flujo sanguíneo renal (FSR); ritmo de filtración glomerular (RFG); fracción de filtración; resistencia vascular renal (RVR); volumen urinario (VU); osmolaridad plasmática y urinaria; depuración osmolar, depuración de agua libre y depuración de sodio y de potasio; sodio y potasio plasmáticos; excreción urinaria y fraccionaria de sodio y potasio y temperatura rectal. Estos atributos fueron evaluados en cuatro momentos: 30 (M1), 60 (M2), 90 (M3) y 120 (M4) minutos después del inicio de la infusión de para-aminohipurato de sodio y creatinina (inicio de la experiencia). En el momento 2, en el grupo G1 fue dada una inyección intra-arterial de solución fisiológica a 0,9\% $(1,24$ $\mathrm{mL} . \mathrm{kg}^{-1}$ ), y en el grupo $\mathrm{G} 2$ fue inyectado contraste radiológico $\left(1,24 \mathrm{~mL}^{\mathrm{kg}} \mathrm{H}^{-1}\right)$ por la misma vía.

RESULTADOS: El grupo G1 presentó aumento de la FC, del FPER, del FSR, de la osmolaridad plasmática, de la depuración de sodio y de la excreción urinaria de sodio; presentó aún disminución de la osmolaridad urinaria, del potasio plasmático, de la depuración de potasio y de la temperatura rectal. En el grupo G2 ocurrió aumento de la FC, de la RVR, del VU, de la depuración osmolar, de la depuración de sodio y de la excreción urinaria y fraccionaria de sodio; ocurrió también reducción del (a): hematócrito ritmo de filtración glomerular, fracción de filtración, osmolaridad urinaria, depuración de agua libre, sodio y potasio urinarios, potasio plasmático y temperatura rectal.

CONCLUSIONES: En este estudio, se concluye que, la inyección intra-arterial del contraste radiológico causó efecto bifásico en la función renal. Inicialmente, provocó aumento de la diuresis y de la excreción de sodio, pero, posteriormente, hubo empeoramiento de las condiciones hemodinámicas $y$, consecuentemente, de la función renal, con aumento de la resistencia vascular renal y disminución del ritmo de filtración glomerular. 